\title{
Telmisartan protects chronic intermittent hypoxic mice via modulating cardiac renin- angiotensin system activity
}

Wanyu Wang ${ }^{1}$, Ailing Song ${ }^{2}$, Yiming Zeng ${ }^{3 *} \mathbb{D}$, Xiaoyang Chen ${ }^{3}$, Yixiang Zhang ${ }^{3}$, Yonghong Shi ${ }^{1}$, Yihua Lin ${ }^{1}$ and Wen Luo ${ }^{1}$

\begin{abstract}
Background: To explore the effects of chronic intermittent hypoxia $(\mathrm{ClH})$, which mimics sleep apnea syndrome, on the cardiac renin angiotensin system (RAS), and to investigate the cardiac protection of an angiotensin receptor blocker (ARB)telmisartan (TERT) against $\mathrm{ClH}$.

Methods: 32 healthy male C57B6J mice were randomly divided into $\mathrm{ClH}$, ARB, blank and air control groups. $\mathrm{ClH}$ lasted for 12 weeks. Cardiac angiotensin converting enzyme (ACE), angiotensin converting enzyme 2 (ACE 2) and angiotensin II (Ang II) were evaluated by immunohistochemistry. Myocardial apoptosis were assessed by TUNEL assay and myocardial cell ultrastructure were observed under transmission electron microscope.
\end{abstract}

Results: Cardiac ACE expression was higher in the CIH group than in blank and air control groups, which was decreased with TERT treatment. TERT treatment elevated the expression of cardiac ACE 2 and Ang II compared with $\mathrm{CIH}$ group. Myocardial cell and capillary endothelial cell apoptosis, mitochondrial injury were most severe in $\mathrm{ClH}$ groups, which were mitigated with TERT treatment.

Conclusions: $\mathrm{CH}$ changes the expression of cardiac ACE, ACE2 and Ang II, which may cause myocardial damage. TERT protects mice from CIH-linked cardiac damage via modulating the activity of RAS in the hearts.

Keywords: Chronic intermittent hypoxia, Sleep apnea syndromes, Telmisartin, ACE, ACE 2, Ang II, Myocardial apoptosis; myocardial cell ultrastructure

\section{Background}

It has been well documented that the incidence of sleep apnea syndromes (SAS) is high in the general population and that SAS may lead to cardiovascular disease (CVDs) [1]. Chronic intermittent hypoxia $(\mathrm{CIH})$ is one of the distinctive pathophysiological features of SAS, and is implicated in the development of SAS-associated CVDs. One of the mechanisms by which CIH promotes CVDs is the activation of the renin-angiotensin system (RAS) [2], a well-known contributor to the pathogenesis of CVDs. However, a key component of RAS, angiotensin converting enzyme 2 (ACE 2), inhibits RAS and exhibits

\footnotetext{
*Correspondence: zeng_yi_ming@126.com; 13599513293@163.com ${ }^{3}$ Department of Pulmonary and Critical Care Medicine of the Second Affiliated Hospital of Fujian Medical University, Sleep and Breathing Disorders Research Institute of Fujian Medical University, No.34 Zhongshan North Road, Licheng District, Quanzhou 362000, Fujian, China

Full list of author information is available at the end of the article
}

vasodilatatory and anti-proliferative functions [3]. Currently, the effects of ACE 2 on the cardiovascular system have been investigated in diabetic animal models. However, the expression and function of ACE 2 in the hearts of $\mathrm{CIH}$ animals has not, to date, been explored. Previously, we reported that we successfully generated a $\mathrm{CIH}$ mouse model and studied the potential mechanisms by which $\mathrm{CIH}$ impaired cardiovascular function [4]. In our published work, we also investigated the protective effects of an angiotensin receptor blocker (ARB), telmisar$\tan$ (TERT), that both has vasodilatory and anti-oxidative activity and is widely used clinically as an antihypertensive [5-7]. We found that $\mathrm{CIH}$ increased oxidative stress in the mouse hearts, as evidenced by increased levels of 8-hydroxy-2'-deoxyguanosine/8-hydroxyguanosine (8-OHdG/8-OHG), malondialdehyde (MDA), and NADPH oxydase p47, which were 
suppressed by TERT treatment [4]. In the present study, we investigated the mechanisms by which $\mathrm{CIH}$ affects the RAS. Furthermore, we explored whether TERT could protect against $\mathrm{CIH}$-induced cardiac damage via modulating the activity of the RAS. Our goal was to elucidate the mechanisms by which TERT, and more generally, $\mathrm{ARB}$, may act in the treatment of SAS.

\section{Methods}

The experimental protocol was approved by Laboratory Animal Ethics Committee of the First Affiliated Hospital of Xiamen University. CIH, ARB treatment, blank control and air control groups, as described previously [4].

A total of 32 healthy male C57B6J mice, with a body weight between 20 and $25 \mathrm{~g}$, were provided by the Laboratory Animals Center, Chinese Academy of Sciences, Shanghai (License number: SYXK (Fujian) 2008-0001). A self-made intermittent hypoxia box, an air control box, and a gas control system to control the gas cycle from oxygen and nitrogen gas to air were used in this study. The S-450 oxygen-detection alarm was purchased from American IST-AIM Company with a sensitivity of $0.1 \%$. Medically compressed oxygen with a concentration higher than 99\%, compressed nitrogen gas with a concentration higher than 99\%, Atman-6500 air pump. The $\mathrm{CIH}$ profiles consisted of alternating room air (21\% oxygen) and $5.5 \%$ oxygen every $120 \mathrm{~s}$. Telmisartan (TERT) tablets (Micardis) were provided by the Shanghai Boehringer Ingelheim Company. Rabbit-anti-mouse antibodies against ACE, ACE 2 and Ang II were obtained from the Wuhan Boster Company. PV9001 kit and diaminobenzidine (DAB) coloring reagent kit were obtained from Beijing Zhong Shan Golden Bridge Inc. In Situ Cell Death Detection Kit (11684817910) was obtained from Roche. JEM-2100HC transmission electron microscope with a charge-coupled device camera (JEM-2100HC) operating at $120 \mathrm{Kv}$ was purchased from Japan Electronics Co, Ltd.

\section{$\mathrm{CIH}$ and ARB treatment model}

The experimental mice were randomly divided into four groups (8 per group): CIH, ARB treatment, blank control and air control groups. An intermittent hypoxia system was used to generate the $\mathrm{CIH}$ model. Briefly, the self-made plexiglass box was constructed with an aperture closed by hyaline film and bilateral wells. The production well contained a one-way flap. The gas inside the box had a constant flow to avoid carbon dioxide retention. The gas control system contained a micro-computer chip, control procedures, relays, solenoid valves and monitors. Each gas supply had an individual air ventilation pipe, all of which were controlled by solenoid valve switches. The oxygen concentrations inside the box were tightly controlled. Each episodic hypoxia cycle time was 2 min (i.e. nitrogen $30 \mathrm{~s}$, resting $30 \mathrm{~s}$, oxygen $20 \mathrm{~s}$ and air $40 \mathrm{~s}$ ). Each gas insufflation was shown on the control program monitor. The oxygen concentration in the box was monitored by the oxygen-detecting alarm.

Mice in $\mathrm{CIH}$ and $\mathrm{ARB}$ groups were placed in the intermittent hypoxia box. Intermittent hypoxia lasted for 12 weeks with $8 \mathrm{~h}$ per day. After eight weeks of intermittent hypoxia, the mice in ARB group were intragastrically administered TERT solution, which contained $0.2 \mathrm{~mL}$ normal saline with the TERT concentration $10 \mathrm{mg} / \mathrm{kg} / \mathrm{d}$ [8], once per day for 4 weeks. In the air control group, the control system and plexiglass box used was the same as that of the $\mathrm{CIH}$ and ARB groups in order to reproduce the same environment. The cycle was similar to the one used in $\mathrm{CIH}$ and ARB groups in order to produce the same noise but only air was used. The blank control group had no any gaseous interference.

After 12 weeks, mice were sacrificed by heart dissection under anesthesia. Mice were fasted overnight followed by anesthesia with an intraperitoneal injection of $3 \%$ phenobarbital $(30 \mathrm{mg} / \mathrm{kg})$, all mice were weighted. Blood samples were collected through a direct cardiac puncture, and were centrifuged at $3000 \mathrm{~g}$ for $15 \mathrm{~min}$ at $4{ }^{\circ} \mathrm{C}$ while the supernatants (serum) were collected and stored at $-80{ }^{\circ} \mathrm{C}$ for further analysis. Mice were vascularly perfused through the heart with cold $100 \mathrm{mM}$ phosphate buffer ( $\mathrm{pH} 7.4$ ). Tissues were immediately removed and the heart was dissected, and cut into small pieces within $1 \mathrm{~mm}$ cubes. The other part of the apex of the hearts was fixed in $10 \%$ neutral buffered formalin, stored in $70 \%$ ethanol, paraffin embedded and sectioned for subsequent histological analysis and TUNEL assay.

\section{Determination of cardiac ACE, ACE 2 and Ang II, TUNEL assay, transmission electron microscope} Immunohistochemistry with the PV9001/DAB two stage method was used to detect the expression of ACE, ACE 2 and Ang II. The primary antibody was used at a concentration of 1:50. All positive staining was brown-yellow. The average optical density values were calculated by IPP 6.0 software. After dewaxing and rehydration, sections were incubating in proteinase $\mathrm{K}$ $(400 \mu \mathrm{g} / \mathrm{mL})$ for $10 \mathrm{~min}$ at $37{ }^{\circ} \mathrm{C}$. TUNEL staining was then performed using In Situ Cell Death Detection Kit (Roche, 11,684,817,910) according to the instructions. After DAB substrate detection, sections were counterstaining with hematoxylin, mounted under glass coverslip and analyzed under light microscope. TUNEL-positive cardiomyocytes in each group were carefully evaluated under double-blinded conditions. Ten high-power fields $(\times 400)$ were randomly selected and scored, and the percentage of TUNEL-positive cells 
was determined by dividing the numbers of positive-staining nuclei by the numbers of total nuclei of the cells. The samples for transmission electron microscope were fixed with $2 \%$ glutaraldehyde in $0.1 \mathrm{~m}$ PBS overnight at $4{ }^{\circ} \mathrm{C}$. After brief washing with PBS, the samples were post-fixed with $2 \%$ osmium tetroxide and $0.5 \%$ potassium ferricyanide in $25 \mathrm{mM}$ cacodylate buffer at $22{ }^{\circ} \mathrm{C}$, followed by dehydration, infiltration and embedding in Spurr's resin. Thin sections $(70 \mathrm{~nm})$ were made and post-stained with uranyl acetate and lead citrate. Samples were viewed under transmission electron microscope. Images were captured at magnifications of 15,000 40,000.

\section{Statistical analysis}

All of data are presented as the means \pm standard error of the mean unless specified otherwise. One-way analysis of variance (ANOVA) followed by the Bonferroni post-hoc test was used to examine statistical comparisons of the mouse cardiac ACE2, and non-parametric Wilcoxon rank sum test was used to determine the statistical significance of cardiac ACE and Ang II data, the rate of apoptotic cells among these groups. The $P$ values were calculated from two-tailed test. $P<0.05$ was considered significant. Statistical analyses were performed using SPSS 20.0.

\section{Results}

\section{TERT decreased the cardiac expression of ACE but} increased the expression of ACE 2 and Ang II in CIH

Immunohistochemistry was used to determine the expression levels of ACE, ACE 2, and Ang II in the apex of mouse hearts obtained from different experimental groups, which are quantified and presented in Table 1. CIH and ARB groups showed stronger ACE staining (Fig. 1a-d) compared with the two control groups (Fig. 1e-h). Similarly, the expression level of cardiac ACE was significantly higher in the $\mathrm{CIH}$ group compared to that in the two control groups (" $p<0.001$, vs. two controls. Table 1$)$. TERT treatment reduced the level of ACE, although this reduction did not reach statistical significance compared with the
$\mathrm{CIH}$ group. No significant difference in ACE expression was found between blank control and air control groups. In the myocardium of the apex of the heart, Ang II staining was mainly localized in the cytoplasm (Fig. 2a-h), and its expression was higher in $\mathrm{CIH}$ group than in blank and air control groups $\left(^{\#} p<0.05\right.$ vs two control groups). However, TERT treatment significantly increased Ang II levels in the heart compared with other experimental groups. $\left({ }^{\&} \mathrm{p}<0.05\right.$ vs. $\mathrm{CIH})$. No significant difference in cardiac AngII expression was found between blank control and air control groups. ACE 2 positive cells showed a similar cytoplasmic staining pattern in cardiac samples obtained from each experimental group (Fig. 3a-h). The expression level of cardiac ACE 2 was higher in the $\mathrm{CIH}$ group than in blank and air control groups $(+\mathrm{p}$ $<0.05$, Table 1), however, TERT treatment significantly increased ACE 2 expression in cardiac samples $\left({ }^{\$} P<0.001\right.$ vs. $\left.\mathrm{CIH}\right)$. There were no significant differences between blank control and air control groups. Collectively, we conclude that TERT decreased the cardiac expression of ACE but increased the expression of ACE 2 and Ang II in CIH mouse hearts.

\section{Pathological changes in myocardial cells under electron microscope}

Next, we evaluated the pathological changes in myocardial cell and capillary endothelial cells in the four experimental groups under electron microscope. While in both the blank and air control groups the chromatin of myocardial nuclei was uniform and the nuclear membrane was intact, in $\mathrm{CIH}$ group the myocardial nuclei were pyknotic, the nucleolus dissolved and disappeared, and the heterochromatin clustered in the perinuclear region, which showed the apoptosis of myocardial cells in 8/8 CIH cases (Fig. 4a-d). Also, in CIH group, the number of mitochondria decreased, the size was abnormal, the mitochondrial membrane was incomplete, the number of cristae was reduced, and the arrangement was disordered, while in the blank and air control groups, the quantity and size of the mitochondrial are normal and the membrane is intact. The mitochondrial cristae filled

Table 1 TERT decreased the cardiac expression of ACE but increased the expression of ACE 2 and Ang II in CIH

\begin{tabular}{llllll}
\hline Group & $\mathrm{n}$ & Cardiac ACE & Cardiac Ang $\|$ & Cardiac ACE 2 & Apoptotic rate of myocardial cells by TUNEL assay (\%) \\
\hline ClH group & 8 & $0.034900 \pm 0.0130^{\mathrm{a}}$ & $0.095922 \pm 0.0328^{\mathrm{d}}$ & $0.052200 \pm 0.0192^{\mathrm{b}}$ & $53.23 \pm 5.292^{\mathrm{f}}$ \\
ARB treatment group & 8 & $0.025100 \pm 0.0061^{\mathrm{a}}$ & $0.149025 \pm 0.0217^{\mathrm{d} c}$ & $0.072850 \pm 0.0175^{\mathrm{b}} \mathrm{c}$ & $23.32 \pm 2.827$ \\
Blank control group & 8 & $0.004738 \pm 0.0026$ & $0.015019 \pm 0.0070$ & $0.017488 \pm 0.0131$ & $22.39 \pm 3.715$ \\
Air control group & 8 & $0.005238 \pm 0.0026$ & $0.020684 \pm 0.0107$ & $0.029313 \pm 0.0136$ & $23.72 \pm 2.857$ \\
\hline
\end{tabular}

${ }^{a} p<0.001$ vs. blank and air control groups

${ }^{b} p<0.05$ vs. blank and air control groups

${ }^{c} p<0.001$ vs. $\mathrm{ClH}$

${ }^{d} p<0.05$ vs. blank and air control groups

$\mathrm{e}_{p}<0.05$ vs. $\mathrm{ClH}$

${ }^{f} p<0.001$ vs. the other three experimental groups 


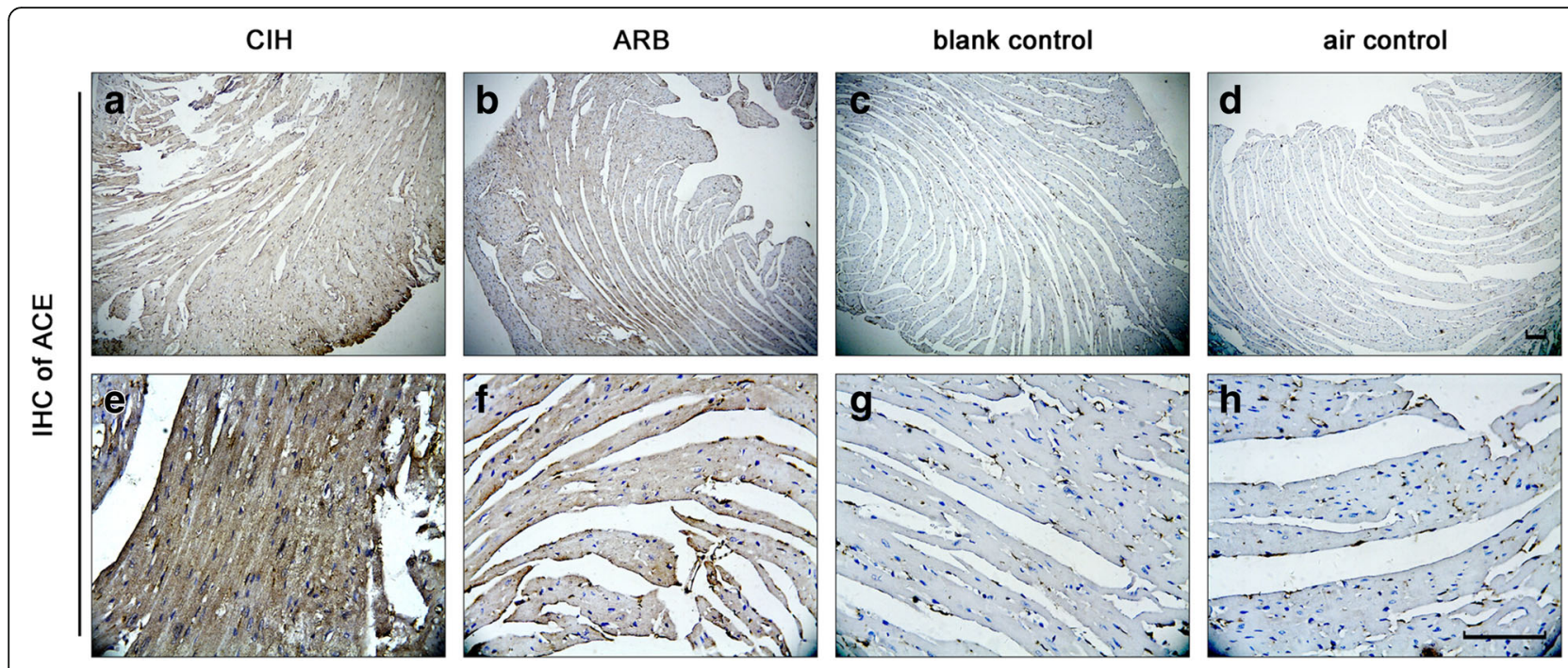

Fig. 1 ACE staining in myocardial cells of mice from four experimental groups. a \& b: CIH group; $\mathbf{c} \& \mathbf{d}$ : ARB group; e \& f:blank control group; $\mathbf{g} \&$ $\mathbf{h}$ : air control group. Immunohistochemistry was performed on the cardiac apex sections as described in Materials and methods. CIH and ARB groups showed highest ACE staining in CIH group followed by ARB group compared with the two control groups although this reduction did not reach statistical significance. Magnification: a, c, e and $\mathbf{g : ~ 1 0 0 x ; ~ b , ~ d , ~ f ~ a n d ~ h : ~} 400 x$. scale bar, $100 \mu \mathrm{m}$

the entire cavity of the mitochondrion and were arranged in parallel, regular and abundant in number (Fig. 5a-d). In addition, in $\mathrm{CIH}$ group, the cristae are broken, dissolved, destroyed, flocculent, as well as increased electron density of matrix increases in 7/8 $\mathrm{CIH}$ cases (Fig. 6a-d). The coarse and fine filaments were well arranged and each band was clearly visible in $\mathrm{CIH}$ group, blank and air control groups. Capillary endothelial cell nuclei were pyknotic, and the heterochromatin clustered in the perinuclear region which showed the apoptosis of capillary endothelial cell in 4/8 $\mathrm{CIH}$ cases (Fig. 7a-d) while no capillary endothelial cells were found apoptotic in the blank and air control groups.

The ultrastructural changes observed in the ARB group were in between those of the $\mathrm{CIH}$ group and the blank and air control groups. Visualization of cardiomyocyte and capillary endothelial cell apoptosis is rare under electron microscopy. The quantity and size of the mitochondria were normal with intact membranes. The electron density of the mitochondrial matrix increased similarly to that observed

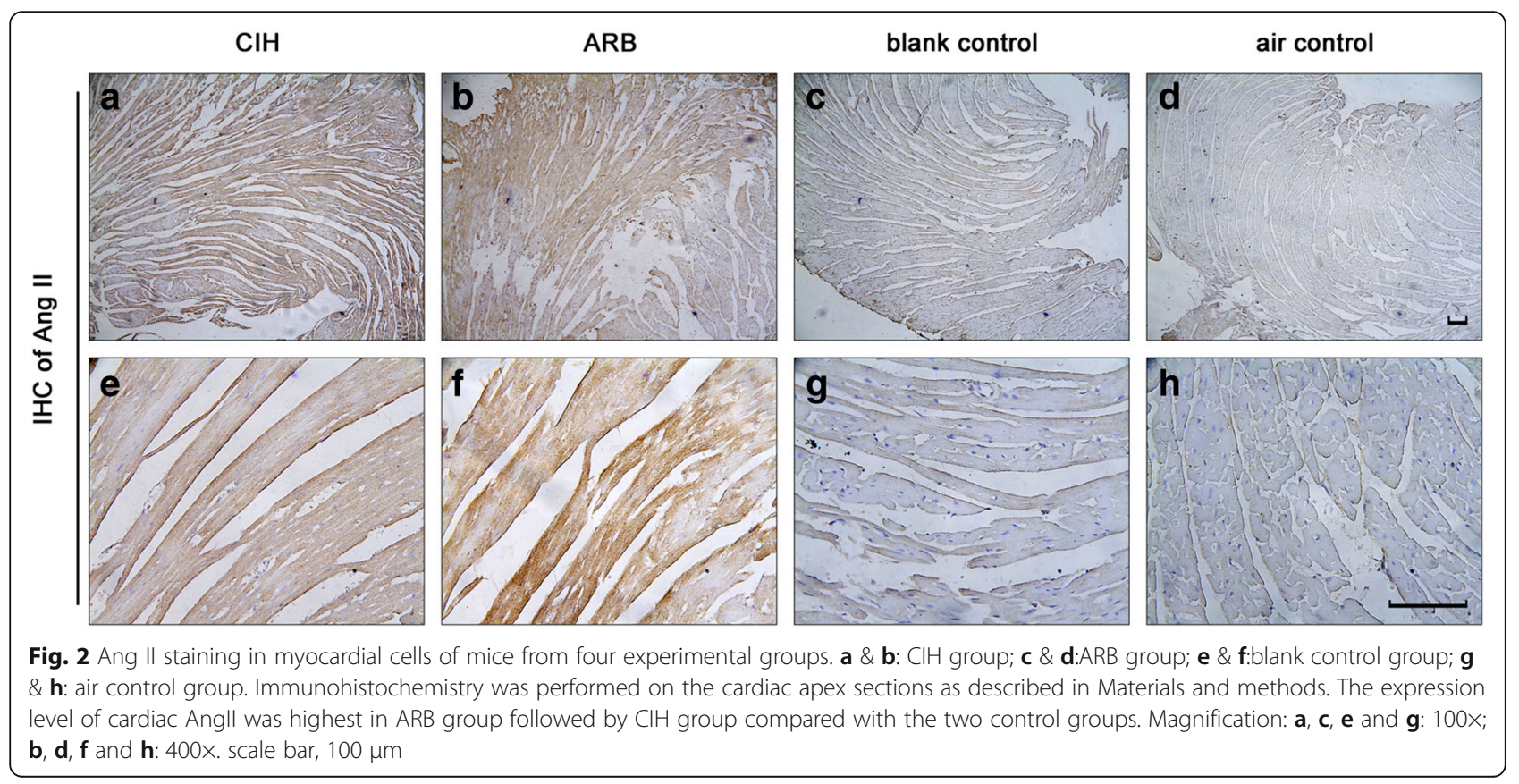




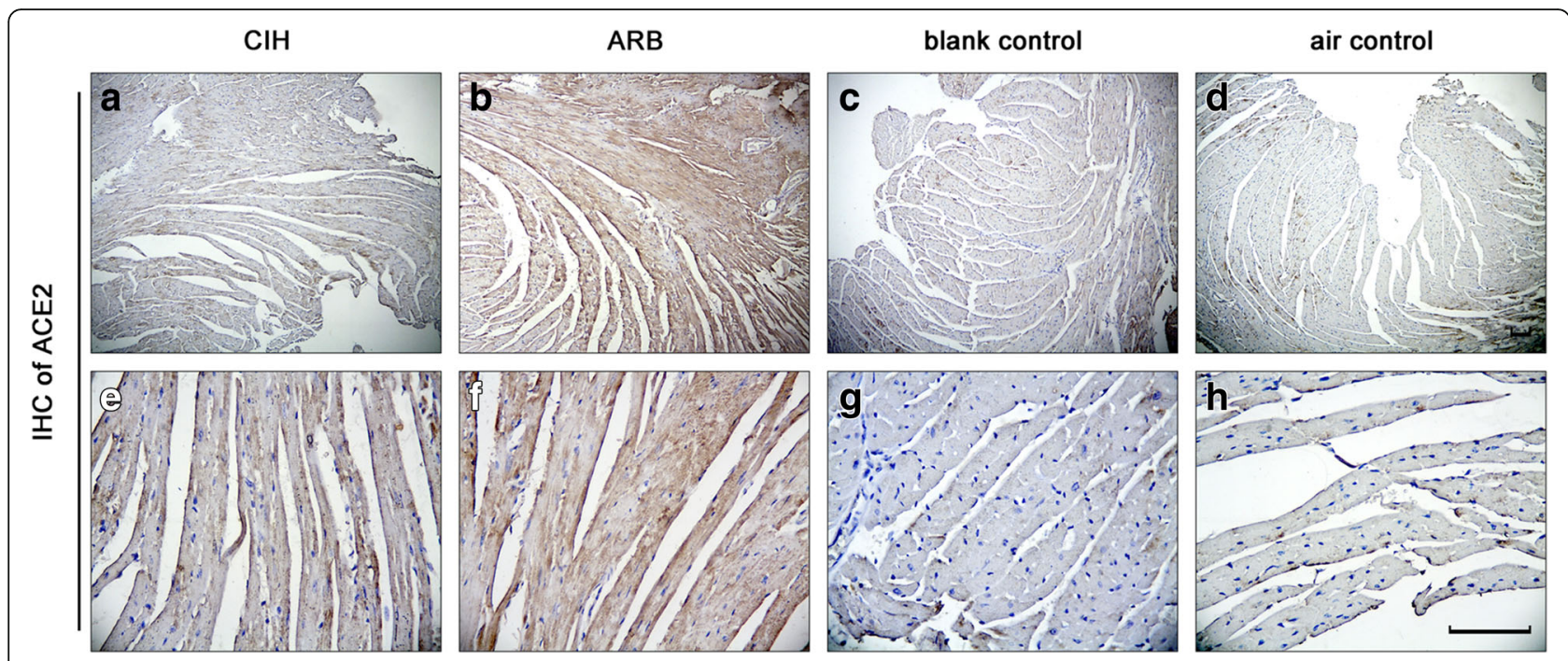

Fig. 3 ACE 2 staining in myocardial cells of mice from four experimental groups. a \& b: ClH group; c \& d:ARB group; e \& f:blank control group; $\mathbf{g}$ $\& \mathbf{h}$ : air control group. Immunohistochemistry was performed on the cardiac apex sections as described in Materials and methods. The expression level of cardiac ACE 2 was highest in ARB group followed by $\mathrm{CIH}$ group compared with the two control groups. Magnification: $\mathbf{a}, \mathbf{c}, \mathbf{e}$ and $\mathbf{g}$ $100 x ; \mathbf{b}, \mathbf{d}, \mathbf{f}$ and $\mathbf{h}: 400 x$. scale bar, $100 \mu \mathrm{m}$

in the $\mathrm{CIH}$ group, however, the number of cristae was normal and well-arranged akin to that of the air and blank control groups. The changes under the electron microscope were closer to blank control and air control groups. Pathological changes were alleviated obviously in ARB group.

We also assessed apoptosis of myocardial cells of mice from these four groups with TUNEL assay. As shown in Fig. 8, while no significant difference in apoptotic rate of myocardial cells was observed between ARB group, blank control and air control groups, which are quantified and presented in Table 1. CIH had a significantly higher apoptotic rate compared with the other three experimental groups $\left({ }^{* \prime \prime} p<0.001\right)$. Thus, CIH impairs myocardial cells structure and increases myocardial cells and capillary endothelial cells apoptosis while these changes can be reversed with TERT treatment.

\section{Discussion}

SAS is closely linked to the development of cardiovascular disease, in which $\mathrm{CIH}$ plays an important role. In our experiment, each intermittent hypoxia cycle lasted approximately two minutes, mimicking 30 times intermittent hypoxia per hour, which was equivalent to severe SAS. Chen et al. [9] studied a $\mathrm{CIH}$ animal model and concluded that the lowest oxygen saturation was approximately $70 \%$, equivalent to severe SAS. In another model, Song et al. [10] also demonstrated that, owing to the gas constant exchange in the box, there was no carbon dioxide accumulation. The partial pressure of carbon dioxide in the blood of mice had no correlation with the intermittent hypoxia cycle, which confirmed that the mice did not rebreathe. These findings eliminated the interference of carbon dioxide retention.

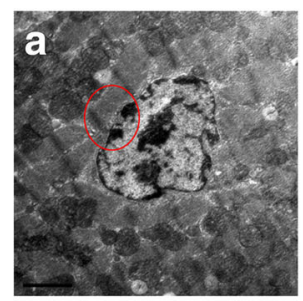

$\mathrm{CIH}$

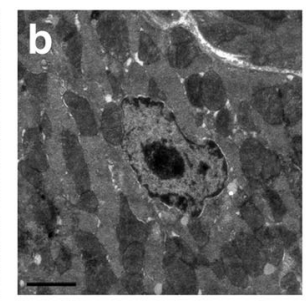

ARB

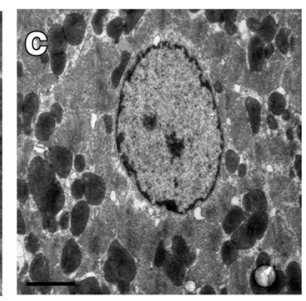

blank control

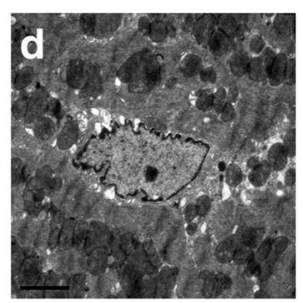

air control

Fig. 4 Pathological changes in myocardial cell nucleus under electron microscope. Ultrastructural examination was performed as described in materials and methods. a In $\mathrm{ClH}$ group, the myocardial nuclei were pyknotic, the heterochromatin clustered in the perinuclear region, which showed the apoptosis of myocardial cells. $\mathbf{b}$ In ARB group, the apoptosis of cardiomyocytes was rare. $\mathbf{c} \& \mathbf{d}$. In blank and air control groups, the chromatin of myocardial nuclei was uniform and the nuclear membrane was intact. Magnification: 15000x, scale bar, $2 \mu \mathrm{m}$ 


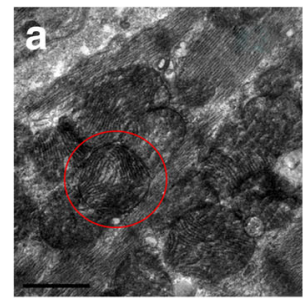

$\mathrm{CIH}$

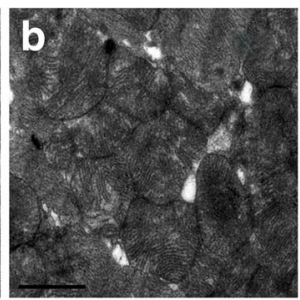

ARB

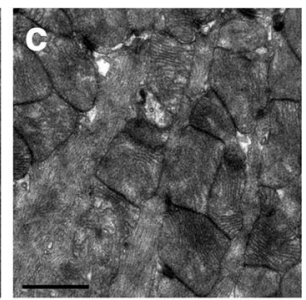

blank control

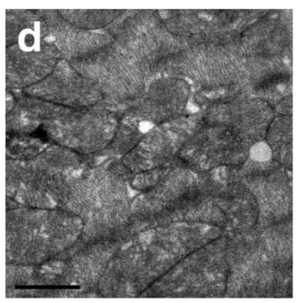

air control

Fig. 5 Pathological changes in myocardial cell mitochondria under electron microscope. Ultrastructural examination was performed as described in materials and methods. a In $\mathrm{ClH}$ group the number of mitochondria decreased, the size was abnormal, the mitochondrial membrane was incomplete, the number of cristae was reduced, and the arrangement was disorder. The cristae were broken, dissolved, destroyed, flocculent, and the electron density of matrix increases. $\mathbf{b}$ In ARB group, the quantity and size of the mitochondrial are normal and the membrane is intact. The electron density of the mitochondrial matrix increased the same as $\mathrm{CIH}$ group while the number of cristae was normal and well arranged. $\mathbf{c} \& \mathbf{d}$ In blank and air control groups, the quantity and size of the mitochondrial are normal and the membrane is intact. The mitochondrial cristae were filled with the entire cavity of the mitochondrion and were arranged in parallel, regular and abundant in number. Magnification: 40000x, scale bar, $1 \mu \mathrm{m}$

$\mathrm{CIH}$ can stimulate hypoxic chemoreceptors in the carotid body, thus activating the systemic sympathetic nervous system and RAS [2], but how CIH affects local RAS and subsequently contributes to the development of CVD remains unknown. In our study, we found that ACE expression was highest in the $\mathrm{CIH}$ group (Fig. 1a, b), suggesting that $\mathrm{CIH}$ could facilitate the expression of ACE in mouse cardiac myocytes in vivo. ACE is the key enzyme of RAS and participates in the systemic and local effects of RAS through endocrine, autocrine and paracrine secretion. The most prominent physiological activity of ACE is to cleave Ang I to Ang II and inactivate bradykinin. Ang II is the product of ACE [11, 12], and ACE can damage the cardiovascular system through production of Ang II. Our study also demonstrated that Ang II expression was higher in the $\mathrm{CIH}$ group (Fig. 2a, b) than in the blank control and air control groups (Fig. 2e-h), indicating that $\mathrm{CIH}$ induced the expression of ACE and its product Ang II which is potentially implicated in CIH-linked CVD. AngII can act on the angiotensin type 1 receptor, and increase inflammation, oxidative stress, apoptosis, and cardiac remodeling, ultimately leading to heart failure. [13] As Ang II can up-regulate the expression of ACE [14], we believe that there was a positive feedback between Ang II and ACE.

ACE 2 is the homolog of ACE, which shares $42 \%$ sequence homology with ACE. The main function of ACE 2 is to cleave Ang I to Ang (1-9) and Ang II to Ang (17). The catalytic activity of cleaving Ang II to Ang (1-7) is 400 times higher than cleaving Ang I to Ang (1-9) [15]. Therefore, the main product of ACE 2 is Ang (17). Ang (1-7) exhibits anti-RAS activity, as well as functioning as a vasodilatation and anti-proliferative agent, suggesting that ACE 2 is the key enzyme in balancing the vasoconstrictive and proliferative effects of Ang II, as well as the anti-proliferative effect of Ang (1-7). In our study, we observed higher expression of ACE 2 in the myocardium of mice from $\mathrm{CIH}$ group (Fig. 3a, b) than that from blank and air control groups (Fig. 3e-h). Since increased ACE 2 levels offer benefits to cardiac structure and function, we speculate that $\mathrm{CIH}$-induced expression of ACE 2 was an adaptive response. Indeed, Zhang $\mathrm{R}$

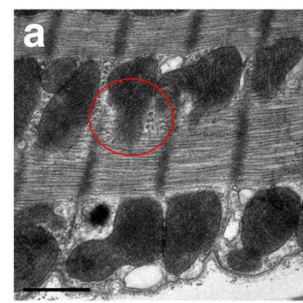

$\mathrm{CIH}$

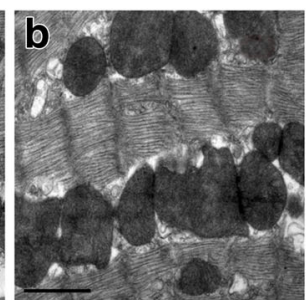

ARB

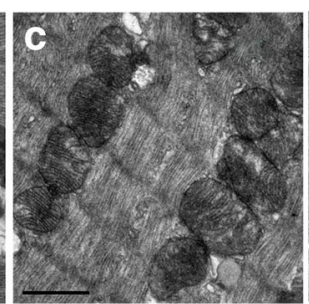

blank control

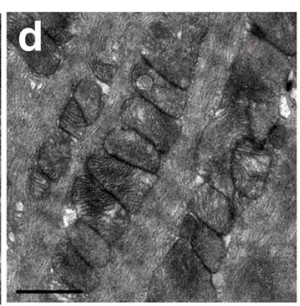

air control

Fig. 6 Pathological changes in myocardial cell mitochondria and myoneme under electron microscope. Ultrastructural examination was performed as described in materials and methods. a In CIH group, the number of mitochondria decreased, the size was unequal and abnormal, the mitochondrial membrane was incomplete, and the electron density of matrix increased. $\mathbf{b}$ In ARB group, the electron density of the mitochondrial matrix increased the same as $\mathrm{ClH}$ group while the quantity and size of the mitochondria were normal and the membrane was intact. c \& d. In blank and air control groups, the electron density of the mitochondrial matrix was nomal, the quantity and size of the mitochondria were normal and the membrane was intact. The coarse and fine filaments were well arranged and each band was clearly visible in four experimental groups (a-d). Magnification: 40000x, scale bar, 1 mm 


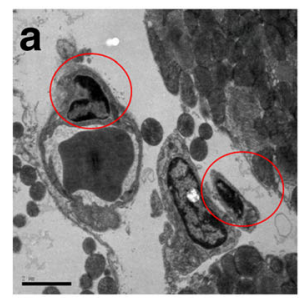

$\mathrm{CIH}$

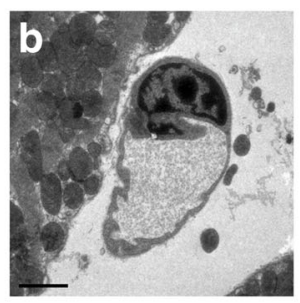

ARB

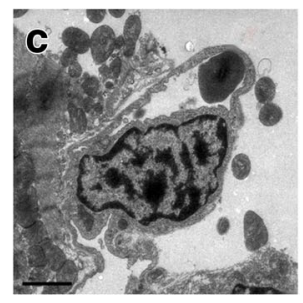

blank control

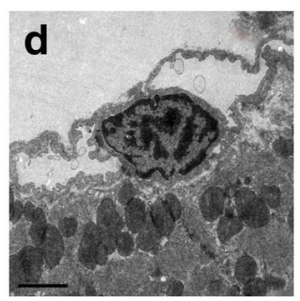

air control

Fig. 7 Pathological changes in capillary endothelial cells under electron microscope. Ultrastructural examination was performed as described in materials and methods. Increased capillary endothelial cell apoptosis was observed in CIH group (a), which was rarely seen in ARB group (b), c \& d. In blank and air control groups the chromatin of capillary endothelial cell was uniform and the nuclear membrane was intact. Magnification: 15000x, scale bar, $2 \mu \mathrm{m}$

[16] demonstrated that ACE 2 mRNA and protein expression increased in the early stages of hypoxia in pulmonary artery smooth muscle cells, but decreased at a later stage, which was accompanied by the accumulation of hypoxia inducible factor- $1 \alpha$ (HIF- $1 \alpha$ ) and Ang II. Thus, it is possible that HIF- $1 \alpha$ downregulates ACE 2 expression through Ang II. In our experiments, $\mathrm{CIH}$ lasted for 12 weeks, and ACE 2 expression remained higher in the $\mathrm{CIH}$ group than in blank and air control groups. Whether local accumulation of HIF-1 $\alpha$ and Ang II at later hypoxic stages was responsible for the downregulation of ACE 2 remains unclear, and requires further study. This may include examining the correlation of the expression levels of myocardial ACE 2 with HIF1 $\alpha$ and Ang II in hypoxic heart tissue for a prolonged $\mathrm{CIH}$ time more than 12 weeks.

In TUNEL assays, we also observed that myocardial cell apoptosis was the most severe in $\mathrm{CIH}$ group. Under

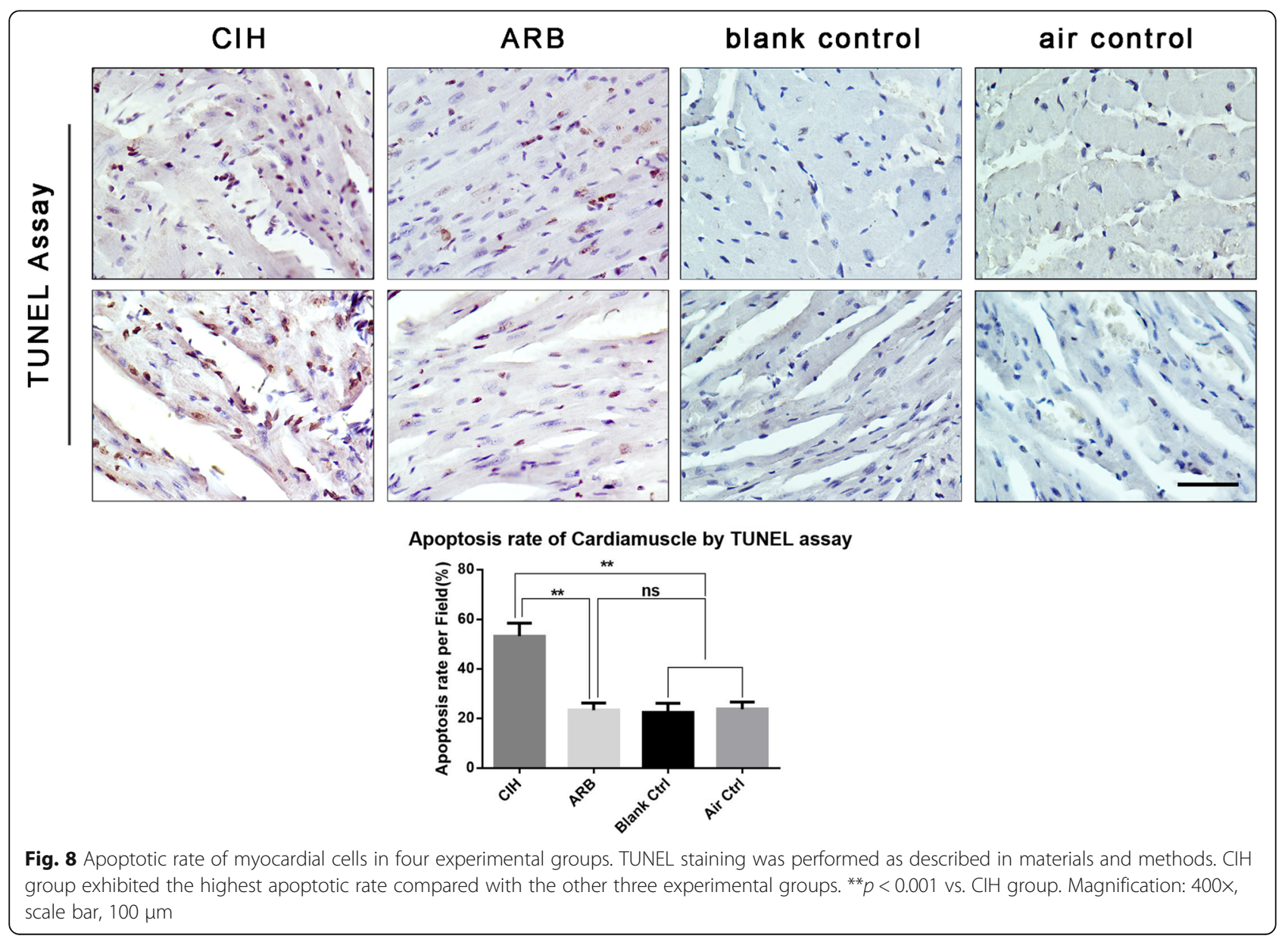


electron microscopy, the apoptosis of cardiomyocyte and capillary endothelial cells were the most serious in $\mathrm{CIH}$ group. We also observed the ultrastructural changes of mitochondria and cristae, indicating that $\mathrm{CIH}$ can also cause mitochondrial damage. Chen et al. reported that the damage of $\mathrm{CIH}$ to mitochondria was related to myocardial apoptosis [17]. Mitochondrial damage is one of the primary mechanisms leading to apoptosis, and hyperactivity of RAS can produce apoptosis in early stages of cardiac disease [18]. Activated RAS can also exacerbate mitochondrial damage and increase apoptosis in endothelial cells, and mitochondria-ROS acts as a central regulator of RAS-mediated cell damage [19].We surmise that $\mathrm{CIH}$ can lead to myocardial damage by elevating the expression of $\mathrm{ACE}$ and Ang II, while the higher expression of ACE2 presents insufficiency of compensation.

Our study also showed that Ang II expression was highest in ARB group (Fig. 2c, d). ARBs can protect the cardiovascular system by blocking the activation of angiotensin type 1 receptor by Ang II, therefore, inhibit the negative feedback for the release of renin. Finally, ARBs increase renin, Ang I, Ang II and Ang (1-7) [20, 21]. Increased Ang II concentrations can interact with the angiotensin type 2 receptor. It is believed that this is mediated by bradykinin and nitric oxide (NO) [22], and the effect of this combination of mediators is both vasodilatory and anti-proliferative, which protects the cardiovascular system. Ang II has been shown to up-regulate the expression of ACE [14], which can be blocked by angiotensin type 1 receptor blocker (ARB) but not by angiotensin-2 receptor blockers, indicating that Ang II-induced expression of ACE was mainly achieved through angiotensin type 1 receptor- (AT-1) activation. In the present study, treatment with TERT, an ARB, reduced the level of ACE induced by $\mathrm{CIH}$, which might alleviate the myocardial damage of $\mathrm{CIH}$. Although this reduction did not reach statistical significance compared with the $\mathrm{CIH}$ group, maximal pharmacological effects of TERT were observed between four to eight treatment weeks, and we only treated mice with TERT for four weeks, which may account for this observation. Therefore, longer treatment with TERT is needed to further clarify if TERT could confer protection against $\mathrm{CIH}$-induced cardiac injury via efficiently suppressing an increase in ACE.

In our study, we also observed that TERT treatment significantly elevated the expression of ACE 2 in the heart, suggesting that this may be one of the mechanisms by which TERT protects against hypoxia-induced heart injury. Previously, Koka et al. [14] showed that Ang II down-regulated ACE 2 expression in HK-2 cell line, which was blocked by the angiotensin type 1 receptor blocker losartan an extracellular signal-regulated kinase (ERK1/2) and p38 MAP kinase-specific antagonist, but not by the angiotensin type 2 receptor blocker PD123319, suggesting that Ang II suppressed ACE 2 expression through angiotensin type 1 receptor-ERK $1 / 2$ and p38 MAP kinase signaling. In the present study, Ang II expression in the hearts was increased in the $\mathrm{CIH}$ group, and this increase was further elevated by TERT treatment. These observations were in line with the TERT-induced increase in ACE 2. But increased Ang II expression did not suppress the expression of ACE 2 in mouse $\mathrm{CIH}$ hearts with TERT treatment, which was most likely due to the blockage of angiotensin type 1 receptor- by TERT.

Under electron microscopy and through TUNEL assay, we observed that the apoptosis of cardiomyocytes and capillary endothelial cells were rare in the ARB group. The ultrastructural changes of mitochondria and cristae induced by $\mathrm{CIH}$ were also alleviated by ARB treatment. We hypothesize that the blockade of the angiotensin type 1 receptor (AT1R) by ARB treatment can elevate the expression of ACE2, thus enhancing the protective effects of ACE 2 and alleviating the myocardial damage induced by ACE and AngII.

Furthermore, TERT, acting as an ARB, can block the effects of Ang II more thoroughly than ACEI, such as Ang II generated from chymotrypsin or other non-ACE pathways. Therefore, TERT may replace ACEI in long-term clinical use to avoid "AngII inhibition escape" phenomenon.

\section{Conclusions}

In conclusion, we demonstrate that $\mathrm{CIH}$ induces the expression of ACE and Ang II in mouse hearts which may impair cardiovascular function. $\mathrm{CIH}$ also increases the cardiac expression of ACE2, which may be a compensatory protection mechanism against $\mathrm{CIH}$. TERT treatment further increased ACE 2 levels in myocardial cells but decreased ACE level, through blocking the interaction between Ang II and AT-1 receptor, impeding the effects of Ang II, thus conferring cardiac protection against $\mathrm{CIH}$.

\footnotetext{
Abbreviations

ACE 2: angiotensin converting enzyme 2; ACE: angiotensin converting enzyme; Ang II: angiotensin II; ANOVA: analysis of variance;

AT1R: Angiotensin type 1 receptor; $\mathrm{CIH}$ : Chronic intermittent hypoxia; $\mathrm{CIH}$ : chronic intermittent hypoxia; DAB: diaminobenzidine;

MDA: malondialdehyde; RAS: renin-angiotensin system; SAS: sleep apnea syndromes; TERT': telmisartan; TUNEL: TdT-mediated dUTP nick end labeling
}

\section{Authors' contribution}

WW, AS, XC and YZ2 performed the research, WW, YS, YL, WL analyzed the data and wrote the manuscript, YZ1 designed the research study, contributed the animal model for the study and revised the manuscript. All authors Approval of the submitted and final versions. 


\section{Funding}

2015 Innovative Project of Fujian Province supported by the Health and Family Planning Commission of Xiamen City, and Innovation Project of Medicine, Health, and Science \& Technology (2015-CXB-36). The funder plays no role in the design of the study; the collection, analysis, and interpretation of data; or in writing the manuscript.

\section{Availability of data and materials}

The datasets used and/or analysed during the current study available from the corresponding author on reasonable request.

\section{Ethics approval and consent to participate}

The experimental protocol was approved by Laboratory Animal Ethics Committee of the First Affiliated Hospital of Xiamen University.

\section{Consent for publication}

Not applicable.

\section{Competing interests}

The authors declare that they have no competing interests.

\section{Publisher's Note}

Springer Nature remains neutral with regard to jurisdictional claims in published maps and institutional affiliations.

\section{Author details \\ 'Pneumology Department of the First Affiliated Hospital of XiaMen University, The first clinical medical college of Fujian Medical University Teaching hospital of Fujian Medical University, Xiamen, China. ${ }^{2}$ Pneumology Department of Wuxi Branch of Rijin Hospital affiliated to Shanghai jiaotong university medical college, Wuxi, China. ${ }^{3}$ Department of Pulmonary and Critical Care Medicine of the Second Affiliated Hospital of Fujian Medical University, Sleep and Breathing Disorders Research Institute of Fujian Medical University, No.34 Zhongshan North Road, Licheng District, Quanzhou 362000, Fujian, China.}

Received: 16 March 2018 Accepted: 25 June 2018

Published online: 03 July 2018

\section{References}

1. Somers VK, White DP, Amin R, Abraham WT, Costa F, Culebras A, Daniels S, Floras JS, Hunt CE, Olson L. Sleep apnea and cardiovascular disease: an American Heart Association/american college of cardiology foundation scientific statement from the American Heart Association Council for high blood pressure research professional education committee, council on clinical cardiology, stroke council, and council on cardiovascular nursing. In collaboration with the National Heart, Lung, and Blood Institute National Center on sleep disorders research (National Institutes of Health). Circulation. 2008;118:1080-111

2. Rey S, Valdes G, Iturriaga R. Pathophysiology of obstructive sleep apneaassociated hypertension. Rev Med Chil. 2007;135:1333-42.

3. Patel SK, Velkoska E, Freeman M, Wai B, Lancefield TF, Burrell LM. From gene to protein-experimental and clinical studies of ACE2 in blood pressure control and arterial hypertension. Front Physiol. 2014;5:227.

4. Wang WY, Zeng YM, Chen XY, Zhang YX. Effect of Telmisartan on local cardiovascular oxidative stress in mouse under chronic intermittent hypoxia condition. Sleep Breath. 2013:17:181-7.

5. Yoo SM, Choi SH, Jung MD, Lim SC, Baek SH. Short-term use of telmisartan attenuates oxidation and improves Prdx2 expression more than antioxidant beta-blockers in the cardiovascular systems of spontaneously hypertensive rats. Hypertens Res. 2015;38:106-15.

6. Bakheit AH, Abd-Elgalil AA, Mustafa B, Haque A, Telmisartan WTA. Profiles of drug substances, excipients, and related methodology, vol. 40; 2015. p. 371-429.

7. Chen XP, Qian LR. The vasodilatory action of telmisartan on isolated mesenteric artery rings from rats. Iranian J Basic Med Sci. 2015;18:974-8.

8. Nagai N, Oike Y, Noda K, Urano T, Kubota Y, Ozawa Y, Shinoda H, Koto T, Shinoda K, Inoue M. Suppression of ocular inflammation in endotoxininduced uveitis by blocking the angiotensin II type 1 receptor. Invest Ophthalmol Vis Sci. 2005;46:2925-31.
9. Chen XY, Zeng YM, Hunag ZY, Tao G, Zheng XZ, Li YJ. Effect of chronic intermittent hypoxia on hypoxia inducible factor-1alpha in mice. Zhonghua Jie He He Hu Xi Za Zhi. 2005;28(2):93-6,

10. Song AL, Zeng YM, CHEN XY. The chronic hypoxia / reoxygenation mice model. International journal of respiratory. Diseases. 2006;26:408-11.

11. Kramkowski K, Mogielnicki A, Buczko W. The physiological significance of the alternative pathways of angiotensin II production. J Physiol Pharmacol. 2006;57:529-39.

12. Igase M, Kohara K, Nagai T, Miki T, Ferrario CM. Increased expression of angiotensin converting enzyme 2 in conjunction with reduction of neointima by angiotensin II type 1 receptor blockade. Hypertens Res. 2008; 31:553-9.

13. Xu J, Carretero OA, Liao TD, Peng H, Shesely EG, Xu J, Liu TS, Yang JJ, Reudelhuber TL, Yang XP. Local angiotensin II aggravates cardiac remodeling in hypertension. Am J Physiol Heart Circ Physiol. 2010:299: H1328-38.

14. Koka V, Huang XR, Chung AC, Wang W, Truong LD, Lan HY. Angiotensin II up-regulates angiotensin I-converting enzyme (ACE), but down-regulates ACE2 via the AT1-ERK/p38 MAP kinase pathway. Am J Pathol. 2008;172: $1174-83$.

15. Zhong Y, Li JF. ANG (1- 7) and its protective effects in hypertension. Chinese J Cardiovasc Dis Res. 2008:6:702-6.

16. Zhang R, Wu Y, Zhao M, Liu C, Zhou L, Shen S, Liao S, Yang K, Li Q, Wan H. Role of HIF-1alpha in the regulation ACE and ACE2 expression in hypoxic human pulmonary artery smooth muscle cells. Am J Physiol Lung Cell Mol Physiol. 2009:297: 631-40.

17. Zeng YM, Cheng XY, Huang ZY, Song AL. Effect of hypoxia/ reoxygenationon the ultrastructure of mice cardiomyocytes and kidney. Fudan University. J Med Sci. 2006;33:84-8.

18. Velez Rueda JO, Palomeque J, Mattiazzi A. Early apoptosis in different models of cardiac hypertrophy induced by high renin-angiotensin system activity involves CaMKII. Journal of Applied Physiol (Bethesda, Md : 1985). 2012:112:2110-20

19. Chen F, Chen B, Xiao FQ, Wu YT, Wang RH, Sun ZW, Fu GS, Mou Y, Tao W, $\mathrm{Hu}$ XS. Autophagy protects against senescence and apoptosis via the RASmitochondria in high-glucose-induced endothelial cells. Cell Physiol Biochem. 2014:33:1058-74.

20. Ferrario CM, Trask AJ, Jessup JA. Advances in biochemical and functional roles of angiotensin-converting enzyme 2 and angiotensin-(1-7) in regulation of cardiovascular function. Am J Physiol Heart Circ Physiol. 2005; 289:H2281-90.

21. Ferrario CM, Jessup J, Chappell MC, Averill DB, Brosnihan KB, Tallant EA, Diz $\mathrm{DI}$, Gallagher PE. Effect of angiotensin-converting enzyme inhibition and angiotensin II receptor blockers on cardiac angiotensin-converting enzyme 2. Circulation. 2005;111:2605-10.

22. Johren O, Dendorfer A, Dominiak P. Cardiovascular and renal function of angiotensin II type-2 receptors. Cardiovasc Res. 2004;62:460-7.

\section{Ready to submit your research? Choose BMC and benefit from:}

- fast, convenient online submission

- thorough peer review by experienced researchers in your field

- rapid publication on acceptance

- support for research data, including large and complex data types

- gold Open Access which fosters wider collaboration and increased citations

- maximum visibility for your research: over $100 \mathrm{M}$ website views per year

At BMC, research is always in progress.

Learn more biomedcentral.com/submissions 